\title{
Sperm motility is enhanced by Low Level Laser and Light Emitting Diode photobiomodulation with a dose-dependent response and differential effects in fresh and frozen samples
}

\author{
C. Philip Gabel ${ }^{1}$, James Carroll ${ }^{2}$, Keith Harrison ${ }^{3}$ \\ 1: Coolum Physiotherapy \\ 2: Queensland Fertility Group, Brisbane, Australia \\ 3: THOR Photomedicine Ltd, UK
}

\begin{abstract}
Background: The effects of Low Level Light Therapy (LLLT) on cellular function arise predominantly from stimulation of ATP production and reduction of oxidative stress. These effects are dose dependent and a function of beam irradiance and irradiation time. Human sperm motility has been shown to increase with LLLT irradiation. The objective of this study was to investigate the effects of laser and Light Emitting Diode (LED) LLLT photobiomodulation on human spermatozoa motility and DNA integrity.

Methods: An in-vitro controlled trial was performed within an IVF clinic laboratory using three human semen specimens, one fresh and two frozen. Sperm were exposed to light from a GaAlAs single laser (810 $\mathrm{nm} 200 \mathrm{~mW}$ ) and an LED cluster $(660 \mathrm{~nm}$ and $850 \mathrm{~nm}$ total power $2 \mathrm{~W}$ ) for various irradiation times. Sperm motility for the test and control aliquots was assessed using a SQA-IIB analyser, but fertilizing ability was not. Sperm chromatin integrity was tested using the Sperm Chromatin Structure Assay.

Results: The Sperm Motility Index and Total Functional Sperm Count increased up to four fold compared to controls with inhibitory effects observed at higher doses (longer irradiation times). The maximum effect varied with irradiance and irradiation time and whether the sample was fresh or frozen.

Discussion: Human sperm motility is modified by exposure to LLLT and this motility modification is dependent upon beam irradiance and irradiation time as well as the condition of the sample. A higher stimulatory dose provides a rapid increase in motility that is short in duration, while a lower stimulatory dose provides a slower increase in motility. An inhibitory does causes reduced motility. Future research could consider animal models, such as the mouse, to test fertilization capacity and the safety of resulting fetuses.
\end{abstract}

Key words: Laser Photobiomodulation • Experimental Research • Effect of Lasers in Tissue • Light Emitting Diode (LED) $\cdot$ Low-Level Laser Therapy

\section{Introduction}

Human infertility affects approximately $15 \%$ of couples trying to conceive and around $10 \%$ of couples require the use of assisted reproductive technologies to succeed ${ }^{1)}$. Male factor infertility accounts for $50 \%$ of couple infertility and sperm motility is a significant contributing cause ${ }^{22}$. The basis for human infertility is evenly attributable to both partners. Male infertility is manifested by oligosper-

\footnotetext{
Addressee for Correspondence:

Philip Gabel

Coolum Physiotherapy, PO Box 760 Coolum Beach, 4573

Qld, Australia,

Tel 61754739614

cp.gabel@bigpond.com
}

mia (sperm density $<20$ million sperm $/ \mathrm{ml}$ ), asthenozoospermia $(<50 \%$ showing progressive motility) and teratozoospermia $(<15 \%$ having normal morphology). However regional and racial variations are present with potential environmental and genetic influencing factors ${ }^{3)}$. Few treatments' are available to correct sperm defects. Improving sperm motility is consequently a key aspect in maximising fertilising capacity ${ }^{2,4)}$. Spermatozoa are highly influenced by the degree of activity within the sperm cation channel, CatSper, which is vital to cAMP-mediated $\mathrm{Ca}^{2+}$ influx in sperm, and subsequent sperm motility essential for fertilising capacity. In addition, sperm motility

Received date: February 2nd, 2018 Accepted date: March 27th, 2018 
is directly influenced by the availability of ATP 5,6). Similarly, the availability of appropriate energy substrates is critical. This indicates a synergistic effect of the motility-stimulating component of the sperm extracts with the available ATP.

Photobiomodulation, through the use of low level laser therapy (LLLT) and light emitting diode (LED) devices, typically in the range $1 \mathrm{~mW}-500 \mathrm{~mW}$, is well documented for improving the production of ATP through the respiratory chain of the mitochondria ${ }^{7,8}$. These light forms also influence the cell membrane lipid bipolar layer affecting ion channel activity and viability ${ }^{9,10)}$. With the knowledge of these sub-cellular actions, and that LLLT has no known harmful effects when applied to tissue ${ }^{11)}$ we postulate that it may have a safe, positive effect on human sperm motility and subsequent oocyte fertilising capacity both in vivo and in vitro.

The specific action of LLLT on sperm has been predominantly assessed through animal models. The positive studies on the effects found in human spermatozoa are limited ${ }^{12,13}$. Extrapolation of the findings from the animal models provided potential insight into the effects of LLLT on human sperm and possible implications for the enhancement of fertility through improved sperm motility. This has included the LLLT effect on enhanced calcium transport and binding by sperm plasma membranes ${ }^{14)}$, the induction of the acrosome reaction ${ }^{15}$ ), an increase in the longevity of motility ${ }^{16)}$ and in frozen animal sperm an augmentation of the survival capability during liquid storage conditions ${ }^{17)}$.

The purpose of this study was to quantify the effects of LLLT on human spermatozoa motility in-vitro and to determine any effect on sperm DNA integrity.

\section{Materials and methods}

Three separate human semen specimens, two frozen two weeks previously from a 32 and a 38 year old, and one fresh from a 37 year old, were collected from volunteer donors with written informed consent and provided within the organization's ethical guidelines. The samples were subjected to LLLT from two continuous output sources at a constant distance of $5 \mathrm{~cm}$ using a THOR Photomedicine system (THOR Photomedicine Ltd, Chesham HP5 1HQ, United Kingdom):

1. A 104 LED cluster $(56 \times 660 \mathrm{~nm}, 10 \mathrm{~mW}$ and $48 \times 850$ $\mathrm{nm}, 30 \mathrm{~mW}$ ), total power $2 \mathrm{~W}$, was projected from a distance of $50 \mathrm{~mm}$ delivering a power density 39.5 $\mathrm{mW} / \mathrm{cm}^{2}$ to the sample. This provided irradiation for periods of 25, 50 and 75 seconds to the first frozen sample and 50,100, 200 and 400 seconds to the fresh sample.

2. A $200 \mathrm{~mW} 810 \mathrm{~nm}$ GaAlAs single divergent laser beam was projected from a distance of $50 \mathrm{~mm}$ delivering a power density $90 \mathrm{~mW} / \mathrm{cm}^{2}$ to the sample. This pro- vided irradiation for periods of 10, 20 and 40 seconds to the second of the frozen samples and 15, 20 and 30 seconds to the fresh sample.

All samples were contained within a four well culture dish each of $1.5 \mathrm{~cm}$ diameter. Sperm motility of test and control aliquots of semen was objectively assessed using a SQA IIB analyzer (MES-Medical Electronic Systems, Migdal Haemek, Israel). The parameters of sperm motility index (SMI) and the total functional sperm count (TFSC) were compared with the calculated various doses of light applied.

The initial experiment utilized two frozen donor semen specimens with the purpose of determining whether photobiomodulation from each light source, Laser and LED, had an effect on sperm motility and the approximate dose for maximum effect.

The second experiment utilized a fresh normal semen specimen and was conducted to validate the optimal light dose from each light source and to determine the longevity of any effect on sperm motility. Two controls were used for this experiment. One was in a well in the test multi-well dish not exposed to light while the second was in the source container of semen.

Sperm DNA integrity was assessed in the fresh control and treated specimen, measured as the DNA fragmentation index (\%DFI) with the Sperm Chromatin Structure Assay (SCSA) ${ }^{18)}$ on a fluorescence activated cell sorter. A very high dose of laser irradiation at $810 \mathrm{~nm}(90$ $\mathrm{mW} / \mathrm{cm}^{2}$ for 1000 seconds) and LED irradiation using a mixed array of $660 \mathrm{~nm} \& 850 \mathrm{~nm}\left(39.5 \mathrm{~mW} / \mathrm{cm}^{2}\right)$ for 400 seconds were compared with the control semen specimen. At no stage was fertilizing ability compared between the test and control aliquots.

\section{Results}

The bio-modulation from the two light sources was consistent across all samples with different degrees of effect. Testing on a frozen semen specimen showed that at 30 minutes post-irradiation, maximal effect on SMI was achieved. Using the 104 LED cluster (660 nm \& $850 \mathrm{~nm}$, $39.5 \mathrm{~mW} / \mathrm{cm}^{2}$ ) just over a four-fold increase in SMI was produced which was achieved with $75 \mathrm{sec}$ exposure (Figure 1), whilst the laser $\left(810 \mathrm{~nm}, 90 \mathrm{~mW} / \mathrm{cm}^{2}\right)$ produced an optimal effect of just below a four-fold increase in SMI (Figure 2) at $20 \mathrm{sec}$ exposure. There was a lesser effect at the longer irradiation time, however the baseline reading was not consistent for the two frozen samples. It was concluded from TFSC results that frozen semen samples were not ideal for experimentation using the SQA analyzer.

With the 104 LED cluster (660 nm \& $850 \mathrm{~nm}, 39.5$ $\mathrm{mW} / \mathrm{cm}^{2}$ ) on a fresh semen sample beneficial effects on SMI were evident at 15 minutes with irradiation times of $50 \mathrm{sec}, 100 \mathrm{sec}$ and $200 \mathrm{sec}$ while a treatment time of 400 
sec had an inhibitory effect. It was found that the best effect on SMI at 30 minutes after exposure came from a treatment time of $50 \mathrm{sec}$ while the longevity of the effect from higher doses diminished more rapidly (Figure 3). Similar results were seen with TFSC. An anomaly was noted in the two control specimens. The motility of that in the original jar diminished faster than that in the unexposed well in the test dish suggesting that the latter had benefited from stimulation by scattered light from neighboring exposed wells.

With the laser $\left(810 \mathrm{~nm}, 90 \mathrm{~mW} / \mathrm{cm}^{2}\right)$ similar beneficial effects on SMI and TFSC were evident at 15 minutes with 15, 20 and 30 seconds of exposure. The best overall longevity of sperm motility, as assessed by both SMI and
TFSC came from the shortest irradiation time (15 sec) (Figure 4). The benefit from higher doses was shorter lived. Again the two controls showed evidence of light scattering in the unexposed well of the multi-well dish compared with the jar control.

Assessment of sperm DNA damage using the SCSA assay revealed no increase in fragmentation from the very high doses of both laser and LED light at approximately 33 times the optimal dose.

\section{Discussion}

This is the first published study that has selected wavelengths from both LED (850 $\mathrm{nm}$ and $660 \mathrm{~nm}$ ) and laser

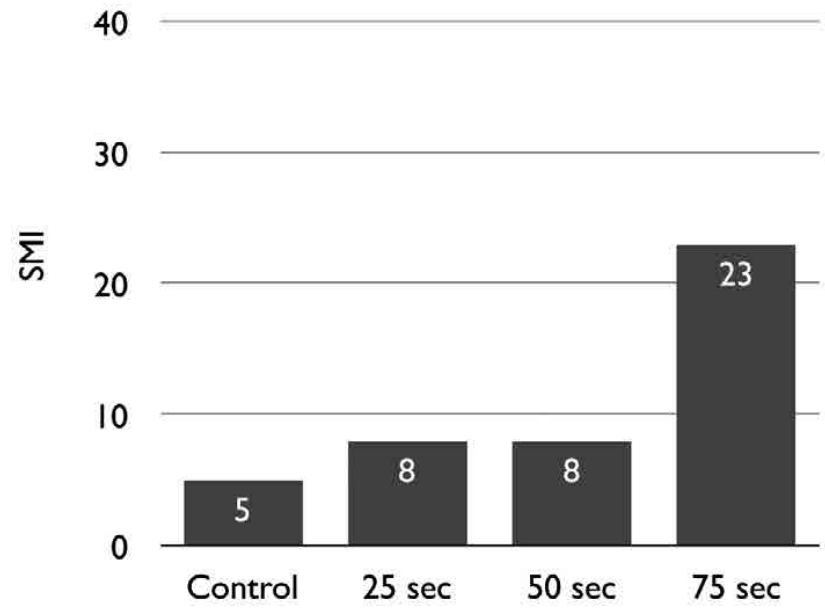

Figure 1: Frozen semen motility, dose response using 104 x LED Cluster Mixed $660 \mathrm{~nm}$ and $850 \mathrm{~nm}$, $45 \mathrm{~mW} / \mathrm{cm}^{2}$

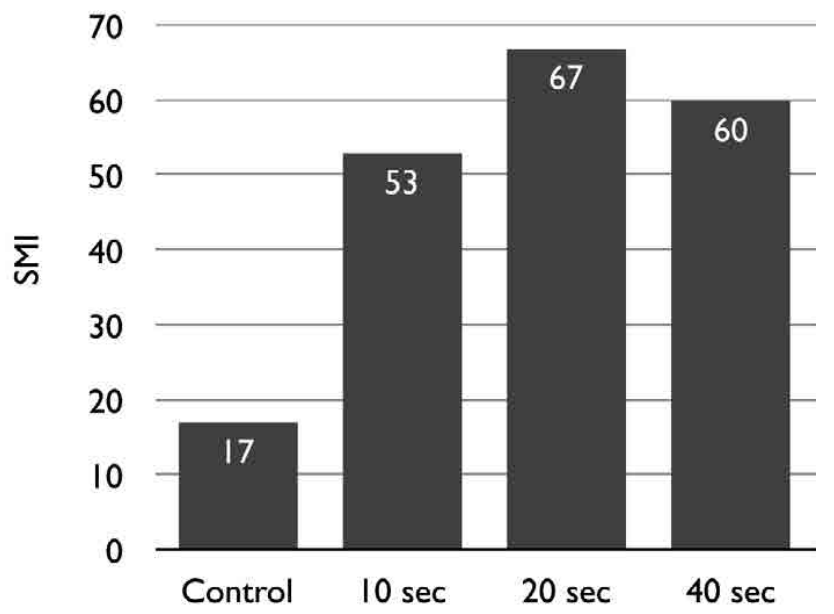

Figure 2: Frozen semen motility, dose response using $810 \mathrm{~nm}$ Laser $90 \mathrm{~mW} / \mathrm{cm}^{2}$

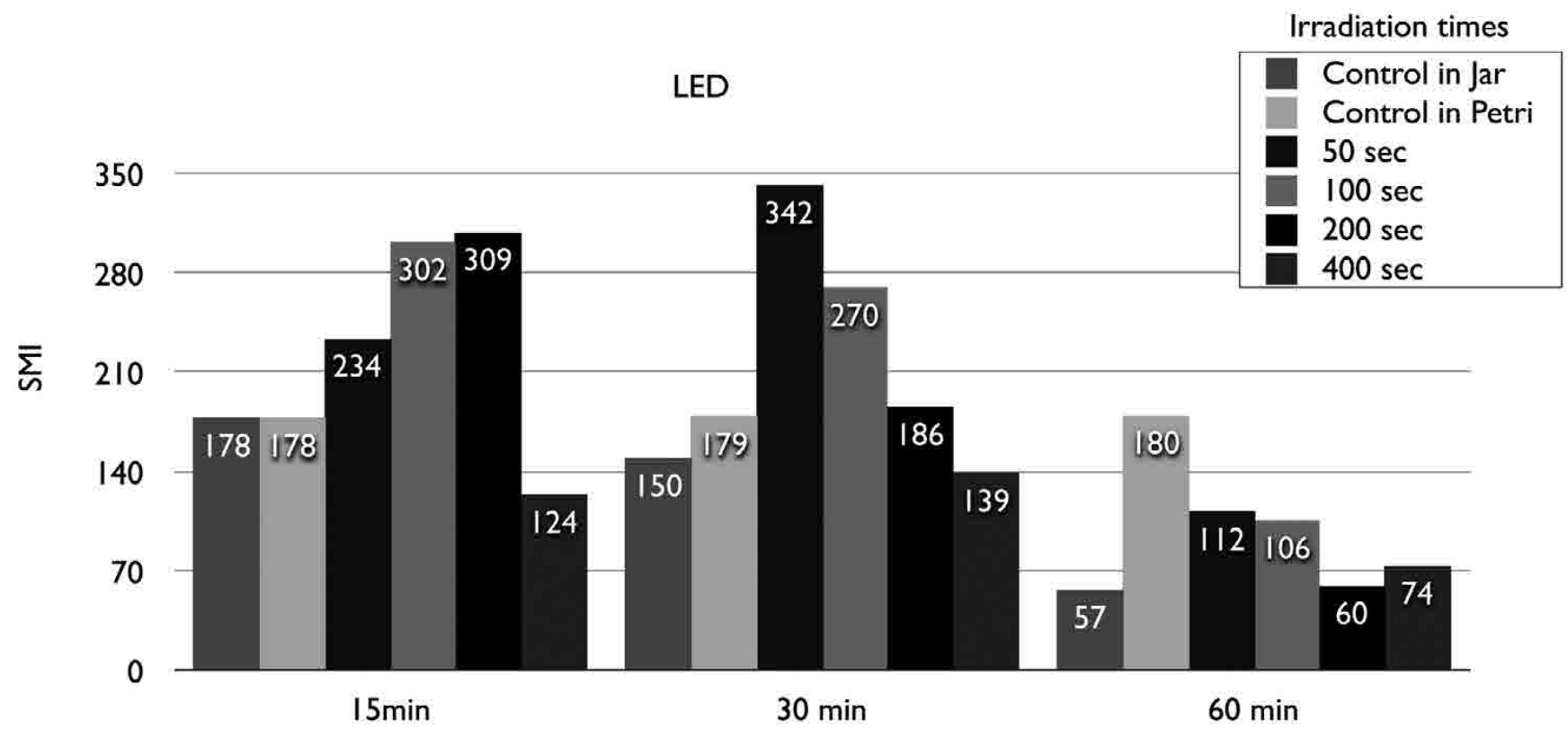

Figure 3: Fresh semen, motility changes over time with different irradiation times, using $104 \mathrm{x}$ LED Cluster Mixed $660 \mathrm{~nm}$ and $850 \mathrm{~nm}, 45 \mathrm{~mW} / \mathrm{cm}^{2}$ 
sources $(810 \mathrm{~nm})$ that replicate those readily available within the clinical setting. These chosen wavelengths also fill some of the knowledge gap in the areas of the effects of sperm motility when exposed to different light wavelengths, as only wavelengths of $905 \mathrm{~nm}^{12)}, 830 \mathrm{~nm}^{13)}$ and $940 \mathrm{~nm}^{19)}$ have previously been used. Other earlier studies have shown LLLT to affect sperm motility but the wavelength and parameters used were not clarified ${ }^{20,21)}$. Of further importance is that the LED source demonstrated conclusively that human spermatozoal motility is modified by exposure to both coherent laser and non-coherent LED light. This finding supports recent research ${ }^{13,12)}$ but is in contrast to earlier work where motility was indicated only in non-motile sperm and that velocity was not affected ${ }^{21}$. Consequently, true laser with its properties of coherence, polarization and monochromaticity ${ }^{22}$ may not be required and LEDs may be an option as has been found in other areas of laser therapy such as wound heal$\operatorname{ing}{ }^{23,8)}$.

The effects of the light irradiation are dependent on both irradiance time and the sample, which is consistent with a biphasic dose response ${ }^{8}$. This capacity for variable response, where low irradiation levels are found to have a greater but variable effect on tissues than higher irradiation levels, is consistent with the Arndt-Schulz curve or "hormesis" ${ }^{24}$ ). There are similar variable dose response findings in previous LLLT research ${ }^{25)}$, including that on sperm motility ${ }^{13,12,26-28)}$. This is different to the findings of earlier work where a linear relationship was suggested between dose and sperm motility ${ }^{20)}$. Different wavelengths may have different effects and coherence, which varies with the LED source ${ }^{8)}$ may also vary the results.

The analysis in this study did not find any damage to sperm DNA integrity by the light irradiation at a very high dose at these wavelengths of $810 \mathrm{~nm}, 850 \mathrm{~nm}$ and $660 \mathrm{~nm}$. This is consistent with previous research at 905 $\mathrm{nm}{ }^{12)}$ but the first report for such corroboration at these wavelengths.

\section{Strengths and Weaknesses}

The study's strengths are that the protocol used is readily adaptable to the clinical environment. The positive findings of both the LLLT and LED indicate that either parameter may be used and that the dose selected will manipulate the velocity and duration of the sperm motility. The differential effect between frozen and fresh sperm indicates that the dose chosen for the latter must compensate for this storage method. The study weaknesses are that the selected devices may not be the optimal for research specific investigation within the in-vitro setting. Improvements may potentially be achieved through the use of homogeneous beams and more accurate irradiation parameters. The SQA analyzer may not be an optimal device for the analysis of motility in frozen semen samples.

\section{Future Research}

There is a clear need to have each of these findings duplicated in further research along with the wavelengths used in both this and earlier studies and different doses in order to determine an optimum dose for maximum

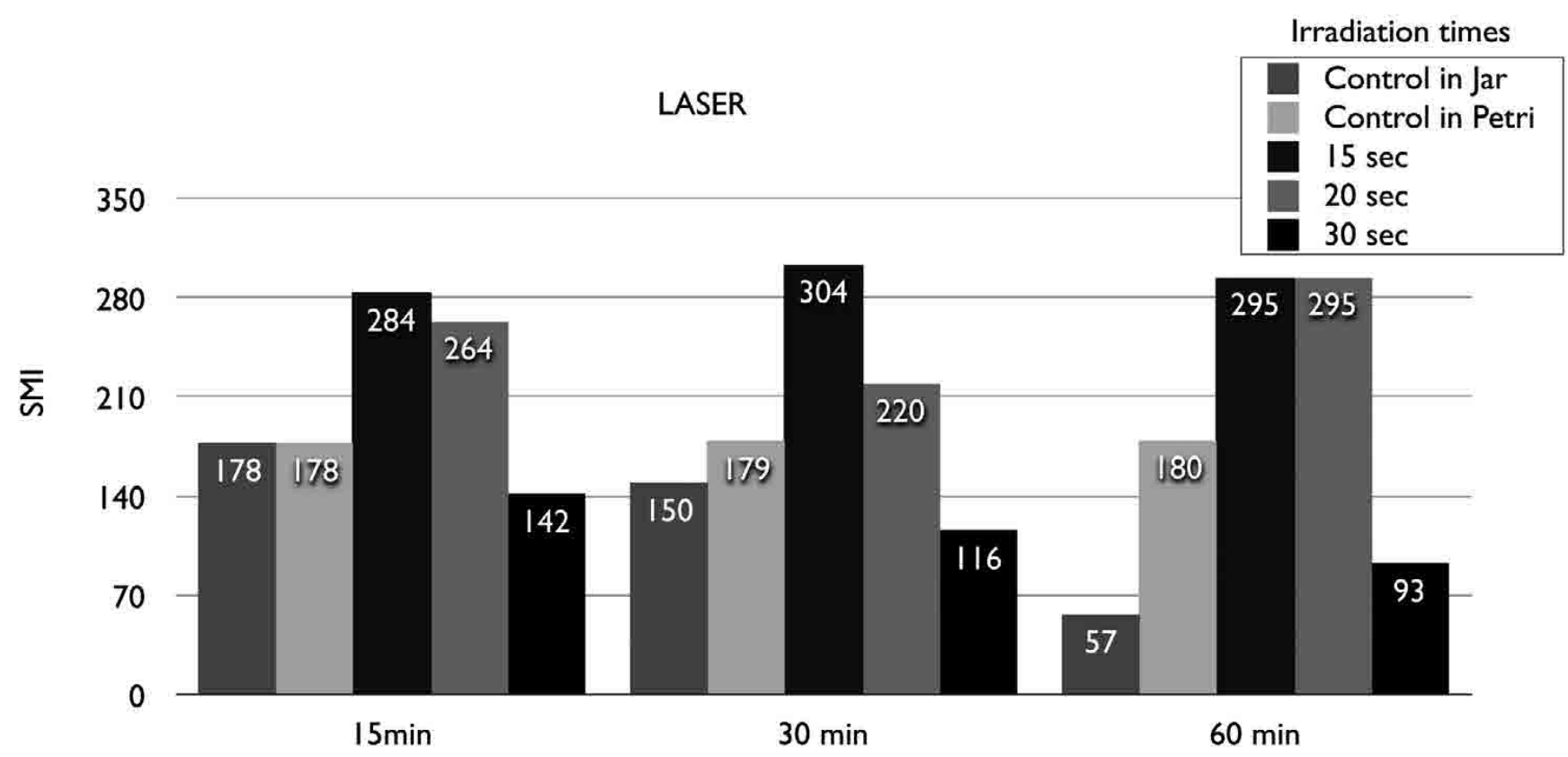

Figure 4: Fresh semen, motility changes over time with different irradiation times, using $810 \mathrm{~nm}$ Laser $90 \mathrm{~mW} / \mathrm{cm}^{2}$ 
motility. Should the in-vitro motility enhancement effect be re-confirmed, along with the absence of any deleterious effects on DNA integrity, in-vivo LLLT treatment of the testes of men with substandard semen could be investigated. This may be for possible short or even longer term benefit to their semen quality. The determination of the velocity change and whether this is linear, as suggested in previous research ${ }^{20)}$, or variable and consistent with the Arndt-Shultz curve, must be clarified. It has been shown that sperm integrity is differentially preserved by varied doses in different animal models with frozen Bovine samples being optimal at 4.0 Joules ${ }^{29)}$ and ram semen quality at $6.12 \mathrm{~J} / \mathrm{cm}^{2} 30$ ). Consequently there is a role for progression to animal models, such as the mouse, to test fertilization capacity and the safety of resulting fetuses.

\section{References}

1: Poongothai J, Gopenath TS, Manonayaki S (2009) Genetics of human male infertility. Singapore Med J 50 (4):336-347.

2: Saharkhiz N, Nikbakht R, Hemadi M (2013) Ketotifen, a mast cell blocker improves sperm motility in asthenospermic infertile men. J Hum Reprod Sci 6 (1):19-22.

3: Ugwuja EI, Ugwu NC, Ejikeme BN (2008) Prevalence of low sperm count and abnormal semen parameters in male partners of women consulting at infertility clinic in Abakaliki, Nigeria. Afr J Reprod Health 12 (1):67-73

4: Ren D, Navarro B, Perez G, Jackson AC, Hsu S, Shi Q, Tilly JL, Clapham DE (2001) A sperm ion channel required for sperm motility and male fertility. Nature 413:603-609.

5: Christen R, Gatti JL, Billard R (1987) Trout sperm motility. The transient movement of trout sperm is related to changes in the concentration of ATP following the activation of the flagellar movement. European Journal of Biochemistry 166:667-671.

6: Ruiz-Pesini E, Diez C, Lapeña AC, Pérez-Martos A, Montoya A, Alvarez E, Arenas J, López-Pérez MJ (1998) Correlation of sperm motility with mitochondrial enzymatic activities. Clinical Chemistry 44:1616-20

7: Karu TI (1989) Photobiology of low power laser effects. Health Physics 56:691-704.

8: Huang YY, Chen AC, Carroll JD, Hamblin MR (2009) Biphasic dose response in low level light therapy. Dose Response 7 (4):358-383.

9: Djordjevic Z (1990) Summary of clinical tests done with the Bioptron Lamp. Bioptron AG, Monchaltorf, Switzerland.

10: Fenyo M (1990) Theoretical and Experimental Basis of Biostimulation by Bioptron. Bioptron AG, Monchaltorf, Switzerland.

11: Tuner J, Hode L (2002) Laser Therapy: Clinical Practice and Scientific Background. Prima Books,

12: Firestone R, Esfandiari N, Moskovtsev SI, Burstein E, Videna GT, Librach C, Bentov Y, Casper RF (2014) The Effects of Low-Level Laser Light Exposure on Sperm Motion Characteristics and DNA Damage. J Androl. 33(3):469-73.

13: Salman Yazdi R, Bakhshi S, Jannat Alipoor F, Akhoond MR, Borhani S, Farrahi F, Lotfi Panah M, Sadighi Gilani MA (2014) Effect of 830-nm diode laser irradiation on human sperm motility. Lasers Med Sci. 29(1):97-104.

14: Lubart R, Freidmann H, Cohen N, Brietbart H Effect of HeNe

\section{Conclusion}

Human sperm motility is modified by exposure to LLLT and this modification is dependent upon beam irradiance and irradiation time as well as the condition of the sample. In vitro enhancement of human sperm motility with LLLT has a number of potential uses in assisted reproductive technologies. Marginally substandard semen could be treated prior to periovulatory timed intrauterine insemination to increase the likelihood of conception. The sperm motility of poorer semen samples could be enhanced prior to in-vitro insemination of oocytes to maximize oocyte fertilization. This may avoid the use of intracytoplasmic sperm injection to obtain fertilization.

laser on calcium signals in sperm cells. In: Proc. SPIE (International Society for Optical Engineering) 1998. pp 45-49.

15: Ocana-Quero JM, Gomez-Villamandos R, oreno-Millan M, Santisteban-Valenzuela JM (1997) Biological effect of helium-neon (He-Ne) laser irradiation on acrosome reaction in bull sperm cells. J Photochem Photobiol 40:294-298.

16: Iaffaldano N, Meluzzi A, Manchisi A, Passarella S (2005) Improvement of stored turkey semen quality as a result of HeNe laser irradiation. Anim Reprod Sci 85:317-325.

17: Iaffaldano N, Rosato MP, Paventi G, Pizzuto R, Gambacorta M, Manchisi A, Passarella S (2009) The irradiation of rabbit sperm cells with He-Ne laser prevents their in-vitro liquid storage dependent damage. Anim Reprod Sci. 119(1-2):1239 .

18: Evenson DP, Jost LK, Marshall D, Zinaman MJ, Clegg E, Purvis K (1999) Utility of the sperm chromatin structure assay (SCSA) as a diagnostic and prognostic tool in human fertility clinics. Hum Reprod 14:1039-1049.

19: Singer R, Sagiv M, Barnet M, Levinsky H, Segenreich E, Fuchs Y, Mendes E, Yehoshua H (1991) Low energy narrow band non-coherent infrared illumination of human semen and isolated sperm. Andrologia 23(2):1814

20: Lenzi A, Claroni F, Gandini L, Lombardo F, Barbieri C, Lino A, Dondero F (1989) Laser Radiation and Motility Patterns of Human Sperm. Arch Androl 23 (3):229-234.

21: Sato H, Landthaler M, Haina D, Schill WB (1984) The effects of laser light on sperm motility and velocity in vitro. Andrologia 16 (1):23-25.

22: Laakso L, Richardson C, Cramond T (1993) Quality of light is laser necessary for effective photobiostimulation? Australian Journal of Physiotherapy 39 (2):87-92.

23: Enwemeka CS (1988) Laser Biostimulation of healing wounds: Specific effects and mechanisms of action. The Journal of Orthopaedic and Sports Physical Therapy 9 (10):333-338.

24: Calabrese EJ (2005) Hormetic dose-response relationships in immunology: occurrence, quantitative features of the dose response, mechanistic foundations, and clinical implications. Crit Rev Toxicol 32 (2-3):89-295.

25: Chow R, Heller GZ, Barnsley L (2006) The effect of $300 \mathrm{~mW}$ $830 \mathrm{~nm}$ laser on chronic neck pain: a double-blind, randomized, placebo-controlled study. Pain 124 (1-2):201-210. 
26: Ban Frangez H, Frangez I, Verdenik I, Jansa V, Virant Klun I. Photobiomodulation with light-emitting diodes improves sperm motility in men with asthenozoospermia. (2015) Lasers Med Sci. 30(1):235-240.

27: Salama N, El-Sawy M. Light-emitting diode exposure enhances sperm motility in men with and without asthenospermia: preliminary results. (2015) Arch Ital Urol Androl. 2015;87(1):14-19

28: Preece D, Chow KW, Gomez-Godinez V, et al. Red light improves spermatozoa motility and does not induce oxidative
DNA damage. (2017) Sci Rep.7:46480.

29: Fernandes GH, de Carvalho Pde T, Serra AJ, et al. The effect of low-level laser irradiation on sperm motility, and integrity of the plasma membrane and acrosome in cryopreserved bovine sperm. PLoS One 2015;10:e0121487.

30: Iaffaldano N, Paventi G, Pizzuto R, et al. Helium-neon laser irradiation of cryopreserved ram sperm enhances cytochrome c oxidase activity and ATP levels improving semen quality. Theriogenology 2016;86:778-84

\section{Conflict of Interest:}

James Carroll is the principal of Thor Photomedicine Ltd., manufacturer of the laser and LED equipment used in the study. 